\title{
Conductas de autocuidado y salud del hombre: el cáncer de próstata como ejemplo
}

\section{Self-care behavior and mens's health: prostate cancer as an example}

\author{
Fernanda da Cruz Bertan, Elisa Kern de Castro \\ Universidade do Vale dos Sinos, Rio Grande do Sul, Brasil.
}

(Rec.: noviembre de 2017 - Acept.: mayo de 2018)

\begin{abstract}
Resumen
El presente artículo tiene como objetivo discutir los factores psicosociales relacionados con el autocuidado orientado a la prevención del cáncer de próstata, su tratamiento y su post-tratamiento, a través de una revisión integrativa de la literatura. El autocuidado presupone un conjunto de actividades aprendidas y orientadas al cuidado de la salud, en las cuales el individuo desempeña un papel activo y responsable relativo a su vida, su bienestar y su salud. Se ha buscado conocer y comprender aspectos psicológicos y comportamentales de los hombres sobre su salud, en la prevención y en las diferentes fases de la enfermedad (diagnóstico, tratamiento y post-tratamiento) presentados en la literatura sobre el cáncer de próstata. Para finalizar, se ha realizado una reflexión sobre la importancia del conocimiento del cáncer de próstata en las diferentes fases de la enfermedad, debido al impacto de esta condición en el público masculino, fundamentalmente, por los problemas relacionados con la vida sexual. Así mismo, se han relevado las intervenciones psicosociales y educativas realizadas por los profesionales de la salud, principalmente psicólogos, para auxiliar en el desarrollo de nuevos estándares de comportamiento y actitudes relacionadas al autocuidado.
\end{abstract}

Palabras clave: Autocuidado; Cáncer de Próstata; Salud del hombre.

\begin{abstract}
This article aimed to discuss the psychosocial factors related to self-care focused on prostate cancer prevention, its treatment and post-treatment, by means of an integrative literature review. Self-care entails a set of activities learnt and oriented towards health care, where individuals play an active and responsible role in their life, well-being and health. This article sought to know and understand men's psychological and behavioral aspects about their health, during the prevention and different phases of the disease (diagnosis, treatment and post-treatment) presented in literature about prostate cancer. Finally, there was a reflection on the importance of knowledge about prostate cancer during the different stages of the disease due to the impact of this condition among men, especially in their sexual life. Furthermore, psychosocial and educational interventions performed by health professionals, mainly psychologists, emerged as means to assist in the development of new behavioral patterns and attitudes related to self-care.
\end{abstract}

Keywords: self-car; prostate cancer, men's health.

Correspondencia: Fernanda da Cruz Bertan. Universidade do Vale dos Sinos, Rio Grande do Sul, Brasil. email: fernandacbertan@hotmail.com 


\section{Introducción}

La salud del hombre ha tenido un creciente interés en las diferentes áreas de la salud, inclusive en la psicología clínica (Schwarz, Gomes, Couto, Moura, Carvalho, et al., 2012). Esto se debe, en gran medida, a la baja demanda masculina de servicios de salud orientados a la prevención de enfermedades (Martins, Gazzinelli, Almeida \& Modena, 2012; Astudillo, Pinzón, Ospina, Grisales, \& García, 2011) y, consecuentemente, a los altos índices de mortalidad en hombres de todas las edades en la mayoría de las enfermedades crónicas cuando son comparados con las mujeres (Carneiro, Santos, Macena \& Vasconcelos, 2016; Silva \& Silva, 2014; Separavich \& Canesqui, 2013).

Históricamente, se presta menos atención a la salud del hombre que a la salud de la mujer (Couto et. al, 2010). Eso ocurre por varios factores, entre ellos, por la preocupación de la salud de la mujer durante el período fértil, embarazo y puerperio. Además, investigaciones relativas a la salud del hombre resaltan la dificultad en la interacción entre las necesidades de cuidados de la población masculina y la organización de las prácticas de salud, fundamentalmente, en lo que se refiere a la atención primaria (Arruda, Mathias \& Marcon, 2017; Ferro \& Borràs, 2011).

Al hablar sobre salud del hombre, se destacan enfermedades que afectan específicamente a esa población, como es el caso del cáncer de próstata (CaP en adelante). Según el National Cancer Institute (NIH, 2017), éste tumor urológico, considerado el más frecuente en hombres en el mundo, generó una previsión cerca de 161.360 nuevos casos de la enfermedad (9,6\% en EEUU) y 26.730 óbitos, solamente para el año 2017. Para la Organización Mundial de Salud (WHO, 2001) hasta 2020 está previsto un aumento global de las muertes relacionadas con el CaP; cerca de 259.000 muertes de hombres de 70 años o más y 103.000 muertes de hombres entre 60 y 69 años.

La elevada incidencia de diagnósticos de $\mathrm{CaP}$ se justifica por el aumento de la expectativa de vida (Cooperberg \& Chan, 2017) y la diseminación de su rastreo (Damião, Figueiredo, Dornas, Lima \& Koschorke, 2015; Belinelo R.G.S.; Almeida, S.M.; Oliveira, P.P. de; Onofre, P.S. de C.; Viegas, S.M da F. \& Rodrigues, A.B. 2014), además de las altas tasas de sobrevivencia (Astudillo et. al, 2011; Perea et al., 2010), pues la enfermedad suele aparecer con el avance de la edad (Benett \& Badger, 2005) y es tratable cuando es diagnosticada precozmente.

Aspectos comportamentales y psicológicos que incluyen el autocuidado del hombre para la prevención del cáncer de próstata, tratamiento y post tratamiento son necesarios para el desarrollo de estrategias de promoción de la salud del hombre. En este contexto, el presente artículo tiene como objetivo discutir los factores psicosociales relacionados con el autocuidado ante el cáncer de próstata desplegado durante su prevención, tratamiento y en el período de post-tratamiento. Esta discusión es llevada a cabo a través de una revisión integrativa de la literatura.

\section{La salud del hombre y el autocuidado}

Los primeros estudios acerca de la salud de los hombres surgieron a finales de los años setenta en Estados Unidos, con un enfoque en los problemas de salud en términos generales. Estudios relacionados con las tasas de morbimortalidad demostraban que los hombres estaban en desventaja en relación a las mujeres (Cooperberg \& Chan, 2017; Courtnay \& Keeling, 2000). A partir de la década del noventa, se buscó focalizar las singularidades de los hombres en el proceso de salud-enfermedad a partir de una perspectiva relacional de género (Courtnay, 2000).

Los estereotipos de género (arraigados hace siglos en la cultura patriarcal) orientan los constructos sociales de feminidad y masculinidad (Pozzati, R.; Beuter, M.; Rocha, L.S.; Santos, N.O.; Budó, M. de L.D. \& Perlini, N.M.O.G, 2013) con el objetivo de intentar explicar las diferencias entre los sexos de forma biológica, comportamental, actitudinal o de funcionamiento psíquico (Barberá \& Gazzinelli, 2004). Estos estereotipos han influido en el desarrollo de patrones de comportamiento que se diferencian entre hombres y mujeres, y son observados también en el autocuidado y en las prácticas de salud (Poonappa, 2014).

Para la sociedad, el hombre aún es percibido con un cuerpo viril, resistente, necesario para la competencia, el trabajo y el sustento de la familia (Costa-Junior \& Maia, 2009), significados que potencian prácticas de salud basadas en creencias y valores de lo que es ser masculino. Estos constructos sociales describen al hombre como un individuo reacio a pedir ayuda, con dificultades para demostrar sentimientos de vulnerabilidad, incluso con amigos o familia, además de evitar buscar la ayuda de profesionales (Addis \& Mahalik, 2003).

En relación a lo anterior, parece haber una identidad masculina culturalmente asociada a la desvalorización de los servicios de salud y a la poca preocupación por la misma (Astudillo et al., 2011) y el autocuidado. Esto explicaría la negación, omisión u ocultación de las necesidades de cuidados relativos a la salud (Costa-Junior \& Maia, 2009) y la poca búsqueda de ayuda profesional, principalmente, en situaciones estresantes (Diaz \& Yaringaño, 2010; Addis \& Mahalik, 2003).

El concepto de autocuidado es discutido hace, aproximadamente, cuarenta años en la literatura, sin un consenso definitivo (Castro \& Moro, 2012; Silva et al., 2008). Está concebido como una tentativa del individuo de regular posibles factores que afectan a su desarrollo, salud y bienestar (Silva et al., 2008). Así, el autocuidado presupone una actividad aprendida y orientada hacia un objetivo que es el cuidado de la salud (Silva et al., 2008) e involucra responsabilidad (Castro \& Moro, 2012).

Las características relacionadas al autocuidado pueden ser divididas en tres categorías: 1) cuidados personales relacionados a situaciones específicas; 2) capacidad de actuar y tomar decisiones, siendo estas influenciadas por el conocimiento, habilidades, valores, motivación, locus de control y eficacia; y 3) cuidados de la salud relacionados con el control individual (Wikimon \& Whitehead, 2009), en el que el individuo es conceptualizado como agente con un rol activo y responsable de su salud (Castro \& Moro, 2012).

Para gestionar una enfermedad y las condiciones asociadas a ella, las personas necesitan comprender su condición de salud y los tratamientos necesarios, además de reaccionar emocionalmente al ajuste a la enfermedad en su proceso de 
adaptación, inclusive en sus actividades de vida básicas e instrumentales (Galvão \& Janeiro, 2013). A fin de comprender el autocuidado en los hombres, es esencial conocer cuáles son los comportamientos que son reconocidos por esta población y que promueven acciones preventivas y de promoción de la salud.

Es frecuente que los hombres tarden en buscar la ayuda de los servicios de salud frente a los síntomas de alguna enfermedad, a no ser que los síntomas afecten directamente su trabajo (Gomes, Nascimento \& Araújo, 2007). Asimismo los hombres son caracterizados con poca disposición para buscar ayuda al enfrentar problemas en la vida, a diferencia de las mujeres (Addis \& Mahalik, 2003). La postergación de la búsqueda del servicio de salud puede ser justificada por el temor a descubrir que algo está mal. Este aspecto se encuentra primordialmente ligado a la aprensión a los posibles cambios acarreados por la enfermedad en caso de necesidad de tratamiento. Tales cambios, entre otros, son: tensión emocional, incapacidad para el trabajo, problemas socioeconómicos. A estos también se suma la preocupación por el mantenimiento del status de proveedor y protector de la familia (Modena, Martins, Ribeiro \& Almeida, 2013). Por lo tanto, el modelo de cuidado $y$, consecuentemente, de autocuidado de la salud esperado por las personas y los profesionales de la salud se acerca al modelo de cuidado de las mujeres. Es decir, este sector de la población tiene prácticas preventivas de salud regulares y accede frecuentemente a los servicios sanitarios.

\section{El autocuidado en el Cáncer de Próstata}

La próstata es una glándula masculina localizada en la parte inferior del abdomen, situada justo debajo de la vejiga y delante del recto NIH (2017). Esta glándula envuelve una porción inicial de la uretra (Guedes \& Caldeira, 2014) y en ella puede ocurrir una disfunción celular que podrá dar origen al tumor: benigno o maligno (Tonon \& Schoffen, 2009).

Con etiología indeterminada, varios factores (algunos en proceso de investigación) están relacionados con el desarrollo del cáncer de próstata, como son el envejecimiento (edad); el metabolismo hormonal (testosterona); el estilo de vida (sedentarismo); la historia sexual; la exposición a sustancias químicas (Hierro, Cromo, Cadmo, goma y Plomo); la urbanización (humo, coches); los hábitos alimentarios (ingestión de grandes cantidades de grasa de origen animal); la herencia genética (padre y hermano con cáncer de próstata) y la raza negra. Entre estos, los más destacados son hormonales, dietéticos, genéticos y ambientales (Damião et al., 2015; Zacchi, Amorin, Souza, Mitto \& Zandonade, 2014).

El cáncer de próstata ( $\mathrm{CaP})$ no presenta señales claras en las fases iniciales de su desarrollo NIH (2017). El síntoma más común es el aumento del tamaño de la próstata, pudiendo ocurrir la obstrucción de la uretra en fases más avanzadas (Guedes \& Caldeiras, 2014). La falta de síntomas hace que el individuo no busque la orientación de un médico, lo cual acaba retardando el diagnóstico y generando casos de mayor gravedad (Tonon \& Schoffen, 2009).

Cuando es diagnosticado en la fase inicial, el CaP tiene excelentes tasas de sobrevivencia (cinco años o más), llegando a un 98,6\% (2007-2013) en el mundo (NIH, 2017). Las tasas de mortalidad más altas se dan entre hombres afro-americanos, con diagnóstico en etapas más avanzadas o metástasis y edades entre 75 y 84 años (NIH, 2017). Según Cooperberg y Chan (2017), es importante resaltar que tanto la incidencia como la mortalidad varían según las regiones geográficas y las poblaciones, reflejando impactos multifactoriales como la variación genética, la dieta, los estilos de vida, factores ambientales, el acceso a cuidados, la expectativa de vida global y las varias políticas de rastreo, como por ejemplo el uso del Antígeno Específico Prostático (PSA).

Así pues, es esencial estar atento a las características genéticas (Modena et. al, 2013) y a los antecedes familiares de esta enfermedad (Bratt, 2002), además de realizar exámenes de prevención que permitan el diagnóstico precoz. Los principales métodos sugeridos son: el Examen del Toque Rectal (ETR) y el uso del Antígeno Prostático Específico (PSA) (Fernandes, Martins, Cardelli, Marcon \& Ribeiro, 2014), siendo éste último el más utilizado entre los hombres (Perea, M.D.; Castaño-Vinyalsb, G.; Altzibare, J.M.; Ascuncef, N.; Moreno, V.; Tardonh, A.; Polláni, M.; Salaa, M., 2012). El objetivo de estos exámenes es el diagnóstico del CAP en las fases iniciales, siendo más efectivos cuando se combinan (Astudillo et al., 2011) pues permiten mejores pronósticos (Damião et al., 2015).

El ETR es un examen de bajo costo (Belinelo et al., 2014) y evalúa la próstata en su tamaño, forma, consistencia (dureza), sensibilidad (dolorosa, incómoda o asintomática) y el tono del esfínter anal (Tonon \& Schoffen, 2009). Además del diagnóstico, este examen permite la identificación de la etapa del $\mathrm{CaP}$ ( $80 \%$ de los tumores se encuentran en la zona periférica de la glándula prostática). A pesar de su eficiencia (Moscheta \& Santos, 2012), este examen es considerado desagradable y embarazoso por los hombres (Damião et al., 2015), factores que se convierten en obstáculo para su realización (Belinelo et al., 2014).

EI PSA es una proteína del líquido seminal, producida principalmente por el tejido prostático, determinado a través del examen de sangre, es un método muy utilizado en el rastreo y el acompañamiento del CaP (Damião et al., 2015, Modena et al., 2013). No obstante lo anterior, no es conclusivo si es utilizado sólo en el proceso de diagnóstico (Tonon \& Schoffen, 2009), adicionalmente su utilización ha de ser cuidadosa. Se están realizando investigaciones para determinar la contribución del PSA y aclarar el impacto de su utilización en la clasificación y en las tasas de mortalidad del CaP (Cooperberg \& Chan, 2017).

Para detectar la enfermedad precozmente es imprescindible que los hombres adopten comportamientos de autocuidado por medio de métodos de detección precoz del CaP. La prevención permite disminuir la incidencia de los diagnósticos tardíos que tienen un mayor impacto en las tasas de mortalidad. Se nota que la poca adhesión al ETR ocurre por la falta de información y por la relación simbólica del examen, su carácter invasivo (punto de vista físico y emocional), el miedo generalizado entre los hombres (Belinelo et al., 2014) y las barreras que pueden estar relacionadas con los estereotipos de género (Moscheta \& Santos, 2012). 
Autocuidado en la prevención, tratamiento y post tratamiento del cáncer de próstata

La falta de medidas preventivas específicas para el autocuidado en la prevención del CaP dificulta el diagnóstico precoz. Según la WHO (2001), las estrategias de clasificación existen, aunque no todas hayan demostrado eficacia. De esta forma, se percibe que las orientaciones utilizadas son, de manera general, para todos los tipos de cáncer e involucran hábitos saludables, a través de la actividad física, la alimentación equilibrada, el control del peso y la no utilización de drogas (Damião et al., 2015). Estas orientaciones combinadas con medidas de prevención primaria (conocimiento de la enfermedad), cuyo objetivo es limitar la exposición a los agentes causales o factores de riesgo; junto con medidas de prevención secundaria, destinadas a organizar procedimientos con la población para diagnósticos precoces, permitirían mejores pronósticos en casi todos los casos (Tonon \& Schoffen, 2009).

Para la Sociedad Europea de Urología (SEU), la detección precoz está basada en la estrategia de orientación del riesgo individual (Tonon \& Schoffen, 2009) para hombres con historia familiar de cáncer. Por otro lado, la Asociación Americana de Urología (AAU), en su última publicación en 2013, sugiere medidas de rastreo en hombres con edades entre 55 y 69 años o en el grupo de edad entre 40 y 54 años, en el caso de hombres con historia familiar de la enfermedad, de raza negra y de origen latinoamericano o africano. Ambas, SEU y AAU, no sugieren el rastreo en hombres por encima de los 70 años (Belinelo et al., 2014; Junior \& Hachul, 2014). Por su parte, la Sociedad Brasileña de Urología (SBU), en su última directriz en 2011, recomienda exámenes anuales de rastreo para el CaP a través del ETR y la dosificación de PSA para hombres a partir de los 50 años. En los casos de hombres de raza negra con antecedentes familiares (padre, hermano e hijo) se sugiere el rastreo a partir de los 40 años (Belinelo et al., 2014; Miranda, P.S.C.; Còrtes, M. da C.J.W.; Ma2004).

Según la WHO (2002), la detección precoz abarca dos estrategias. Una para personas con señales y síntomas de la enfermedad (diagnóstico precoz) y otra, para personas aparentemente saludables (rastreo). En este contexto, el estímulo a la realización de los exámenes preventivos para el CaP (Belinello et. al, 2014) asociado al conocimiento sobre la evolución de la enfermedad y al acceso a los servicios médicos y de laboratorio son esenciales. Este conjunto de factores permite el diagnóstico de la enfermedad en su fase inicial y, en la mayoría de las ocasiones, los mejores pronósticos (Miranda et. al, 2004).

Las estrategias educativas pueden ser para cualquier individuo -independientemente de la edad y el estatus socioeconómico- una excelente herramienta de transmisión de información sobre cuestiones de salud (Sorensi et. al, 2012), principalmente, sobre el cáncer. El equipo de salud debe ofrecer servicios alternativo cuando detecte que la educación sobre autocuidado es inadecuada (Hasanpour-Dehkordi, 2016).

En este contexto, la prevención del CaP aún demuestra ser insuficiente. En la revisión sistemática conducida por Pedersen, Armes y Rean (2012), se ha identificado el poco conocimiento sobre esta enfermedad y sus exámenes preventivos en diferentes grupos de hombres, principalmente en la raza negra. Miedos y tabús asociados al $\mathrm{CaP}$, junto con la falta de relación de confianza con los profesionales de la salud, pueden tener un impacto en la disposición de esta población a discutir la necesidad de un comportamiento activo de autocuidado, el cual favorecería la decisión de realizar exámenes preventivos como el PSA y el ETR. Estos factores pueden comprometer el comportamiento del autocuidado de los hombres, principalmente en la etnia negra. En este sentido, los esfuerzos por aumentar el acceso a información sobre el CaP y a los servicios médicos disponibles podrían incrementar la percepción del riesgo de sufrir una enfermedad grave, como este tipo de cáncer.

Otro de los factores que podrían influir en la percepción de riesgo personal está relacionado con la historia familiar de cáncer (Bratt, 2002), esto es, la experiencia del padre y/o hermano (principalmente) con diagnóstico de la enfermedad. Esta información podría influir en el reconocimiento de los síntomas y consecuentemente en la búsqueda de asistencia médica. A pesar de que la literatura no presenta síntomas claros para el CaP NIH (2017), se sabe que están relacionados con el aparato urinario y cualquier cambio podría activar la percepción del riesgo individual.

Aún con la aparición de síntomas de la enfermedad, los hombres pueden demorar en buscar asistencia médica. Evidencia de lo anterior se puede encontrar en una investigación realizada en el sur del Brasil con 54 hombres con diagnóstico de CaP. Los resultados demuestran que $37 \%$ de los encuestados descubrieron la enfermedad a través de exámenes cuando ya habían aparecido síntomas obstructivos (43\%) e irritativos (40\%). Además, inclusive con la aparición de los síntomas, el $8 \%$ de esta muestra informó haber tardado más de seis años en buscar asistencia médica (Fernandes et al., 2014).

En el caso de hombres con historia familiar de CaP (factor de riesgo para el desarrollo de esta enfermedad) asociada al reconocimiento de posibles síntomas (aumento del tamaño de la próstata, síntomas obstructivos y/o irritativos) y el conocimiento de la enfermedad, es factible pensar que la conjunción de estos tres factores podría aumentar la percepción del riesgo personal. Lo anterior influiría en la búsqueda de cuidados médicos, en el autocuidado y en un posible diagnóstico precoz. Para Bratt (2002), la historia familiar de cáncer puede tener un papel fundamental en su prevención y debería ser el detonante para la búsqueda de informaciones y la realización de exámenes preventivos.

Investigaciones previas demuestran que los hombres poseen poco conocimiento de la enfermedad $y$, principalmente, sobre los exámenes preventivos. Respecto al conocimiento del examen PSA para el rastreo del $\mathrm{CaP}$ en hombres con edades entre 50 y 70 años, se ha observado que las informaciones son escasas y erróneas (Escurriola, Font, Forès, Brunet, \& Torrens, 2008), insuficientes para tomar la decisión de realizarlo como forma de rastreo de la enfermedad. Resultados semejantes han sido puestos de manifiesto en el estudio conducido por Chan et. Al. (2011) que ha constatado la poca discusión y participación en la toma de decisiones por parte de los hombres a la hora de realizar el examen PSA con su médico.

Las dificultades sobre la falta de información acerca del CaP, los exámenes preventivos y las formas de tratamiento afectan a toda la población, independientemente del nivel educacional y socioeconómico. En el estudio realizado por Miranda et al. (2004) focalizado en la realización de prácticas preventivas 
en 135 profesores y médicos, se constató que el 20,7\% de los profesionales participantes de 51 años o más, nunca se habían realizado el ETR o la dosificación de PSA como prácticas de detección precoz del CaP y el 36,2\% nunca se había sometido al ETR. Estos resultados demuestran que aún con un nivel educacional y socioeconómico alto, tampoco es común la realización de exámenes preventivos para el CaP.

En un estudio de revisión sistemática, fueron identificados algunos factores que pueden intervenir en la toma de decisiones respecto a la realización de exámenes preventivos, así como en el desarrollo de comportamientos de autocuidado, tales son: vergüenza de realizar exámenes, la falta de información, el miedo a descubrir la enfermedad o a las consecuencias secundarias del tratamiento como la disfunción sexual y la incontinencia urinaria. Estos aspectos pueden contribuir a la no realización del diagnóstico y del tratamiento precoz (Gomes, Izidoro \& Mata, 2015).

Otros factores como la consciencia sobre el riesgo personal de los hombres que tienen una historia familiar relacionada con esta enfermedad (Chan et al., 2011); diálogo activo con el profesional médico sobre las dudas relacionadas con la enfermedad, su diagnóstico y tratamiento, independientemente del nivel de escolaridad, socioeconómico (Chan et al., 2011) y etnia (Pedersen, V.H.; Armes, J. \& E. Rean, 2012); junto con la desmitificación del ETR, facilitando información de cuándo y cómo realizar este procedimiento, pueden disminuir la incomodidad de los hombres (Mestrino, Gomes, ,Almeida, Almeida, \& Oliveira, 2011).

Para ello, es necesario aumentar los esfuerzos de los profesionales de la salud en la diseminación del conocimiento sobre el CaP, en la tentativa de eliminar "malentendidos", respecto al diagnóstico, tratamiento y acceso a los servicios de salud (Pedersen et al., 2012). Estas medidas pueden mejorar la adhesión al autocuidado, pues se comprende que cuanto mayor es la exposición a las informaciones sobre la enfermedad, mayor es el estímulo para la realización de los exámenes de diagnóstico.

En lo que se refiere al autocuidado en la fase del tratamiento del CaP, éste tiene como objetivo auxiliar al paciente en la adaptación a la condición de enfermo y la búsqueda de la mejor forma de enfrentar las dificultades derivadas de las intervenciones terapéuticas (Moscheta \& Santos, 2012), la prevención de complicaciones y los ajustes de los hábitos de vida (Willians \& Pace, 2009). Al relacionar el autocuidado con el cáncer, han sido identificadas cuatro características: educación, interacción, autocontrol y autoconfianza, asociadas al cliente (autoeficacia, autoestima), al sistema (fuentes adecuadas, redes sociales y factores culturales) y a los profesionales de la salud (participación) (Hasanpour-Dekordi, 2016). Junto con la confirmación del diagnóstico de $\mathrm{CaP}$, es necesario clasificar el tumor (Damião et al., 2015), pues este está considerado el principal factor de pronóstico de sobrevida (clasificaciones clínicas I y II) (Zacchi, S.R.; Amorin, M. H.C.; Souza, M.A. C. de; Mitto, M.H.M. de B. \& Zandonade, E., 2014). Los principales tipos de tratamientos para el CaP son la radioterapia, la prostatectomía radical, la terapia endócrina (Tono \& Schoffen, 2009) o la hormonoterapia (Damião et al., 2015) y la castración (orquiectomía) en casos graves. A este respecto, es necesario que médico y paciente discutan el mejor tratamiento (Damião et al., 2015; Guedes \& Caldeiras, 2014) considerando factores como la expectativa de vida del paciente, la existencia de comorbilidades y efectos colaterales de cada tratamiento.

Según NIH (2017), el 75\% de los hombres tratados con CaP localizado relatan efectos colaterales severos y persistentes, como disfunción sexual, incontinencia urinaria y función intestinal de baja calidad. Recibir un diagnóstico de cáncer puede llevar al paciente a tener problemas socioeconómicos, depresión y aislamiento social (Pinquart \& Dubertein, 2010). Asociado a los efectos colaterales del tratamiento, es necesario el acompañamiento de un equipo interdisciplinario (Damião et. al, 2015). Esta interacción es esencial (Haynes, Bonner \& Pryor, 2010) para un adecuado planeamiento de la salud y debe ser ejecutada de forma colaborativa, con pacientes activos y comprometidos (Bolster \& Manias, 2010), dicho de otro modo, con afectados que ejerzan el autocuidado durante su tratamiento.

En la revisión sistemática sobre los cuidados de enfermería durante la preparación del paciente prostatectomizado y el post-operatorio han sido identificadas las siguientes intervenciones: conductas generales, cuidados del catéter vesical, prevención de infecciones, cuidados en la nutrición e hidratación, retorno a las actividades, cuidados respecto a la higiene, cuidados con la administración de los medicamentos, señales y síntomas esperados en el post-operatorio, señales y síntomas de complicaciones y conducta, orientación sobre ejercicios para la musculatura y cuidados para controlar el dolor. Se ha detectado que la principal estrategia utilizada para el cuidado ha sido basada en la educación, a través de la información oral y escrita (combinadas) para el paciente y el familiar, orientadas a favorecer el cuidado, fundamentalmente, en casa (Mata \& Napoleão, 2010).

Al hacer la relación entre las orientaciones de cuidado y el comportamiento de autocuidado, se espera que en la prostatectomía el paciente con CaP sea, por ejemplo, activo y responsable frente a las orientaciones realizadas de cara al procedimiento. En este caso, el proceso de educación sugiere el conocimiento de la enfermedad, su tratamiento y sus posibles complicaciones; el monitoreo de señales y síntomas que puedan permitir intervenciones (si es necesario), sumado a los ajustes relacionados al proceso de la enfermedad en su vida diaria. En definitiva, se persigue un planeamiento de salud dentro del cual el paciente sea activo en el comportamiento de autocuidado, interactuando y buscando información de forma continua o hasta que las dudas sean respondidas.

La fluctuación de los síntomas es una de las principales dificultades del autocuidado durante el tratamiento, derivada del proceso del avance de la enfermedad (Castro \& Moro, 2012). En este contexto, el auto-monitoreo es una característica asociada al autocontrol y los cuidados personales, siendo esencial para el autocuidado (Wilde \& Gavin, 2007). No obstante, en lo que se refiere al $\mathrm{CaP}$, por afectar la región anatómica responsable de las funciones sexuales, las consecuencias específicas del tratamiento de la enfermedad dan un mayor énfasis al monitoreo de los síntomas relacionados con el aparato urinario, principalmente la incontinencia urinaria, la disfunción sexual y la disminución de la libido, no siendo abordado de forma completa el comportamiento de autocuidado.

Estos factores pueden desencadenar conflictos relacionados con la sexualidad (Moscheta \& Santos, 2012). Al trazar el perfil 
de los hombres con diagnóstico de $\mathrm{CaP}$, se ha identificado que los cambios en la dimensión física han sido de igual importancia que los cambios en la dimensión emocional (Fernandes et al., 2014). Según los autores, aspectos relacionados con la sexualidad tales como la alteración de la erección, la calidad y la frecuencia de la erección y la impotencia completa, derivados del proceso de la enfermedad, han exigido un enfrentamiento emocional significativo por parte de estos pacientes y pueden provocar las peores situaciones de estrés.

De acuerdo con Modena et al., (2013) la recurrencia de cuadros de impotencia y pérdida de la libido que dificultan el acto sexual de la forma que era antes de la enfermedad provoca en los hombres la sensación de la pérdida y/o sustracción de la masculinidad. Considerando que la identidad masculina está asociada al ejercicio activo y constante de su sexualidad, problemas de este orden pueden transformarse en una preocupación constante para el paciente. La enfermedad puede afectar a la autoconfianza y desencadenar sentimientos como miedo, angustia, ansiedad, inutilidad, sensación de vacío. Estas manifestaciones psicológicas afectan significativamente las prácticas de autocuidado (Pinquart \& Duberstein, 2010).

Según Bennet y Badger (2005), profesionales sanitarios no aborda los aspectos emocionales y psicológicos de forma adecuada entre hombres con diagnóstico de $\mathrm{CaP}$, generando pocas evidencias sobre la prevalencia de síntomas depresivos en esta población. A través de un estudio sobre la depresión en hombres, los mencionados autores identificaron la tendencia aparente (de los hombres) a alejarse de las emociones y sentimientos depresivos. En contrapartida, detectaron consecuencias a través de comportamientos autodestructivos (abuso de sustancias químicas, auto negligencia), mal adaptados, desajustados y abusivos, considerados una expresión de los síntomas de la depresión. Para los autores, hombres mayores, casados y con soporte social alto, manifiestan un mayor optimismo, menos preocupación con el funcionamiento físico y menor riesgo clínico para desarrollar depresión.

Según Pedrosa y Polejack (2016), los pacientes con diagnóstico de cáncer manifiestan que la vivencia de la enfermedad los torna más conscientes y activos en relación al autocuidado. En lo que se refiere a los hombres, esta vivencia es como una ruptura con su biografía de vida, llevando a la necesidad de cambios significativos en su identidad y en el ejercicio de la masculinidad. Durante este proceso, enfrentan limitaciones y fragilidades hasta entonces negadas y/o descuidadas, revelando la necesidad de dar un nuevo significado a su papel social (Modena et al., 2013).

Otro factor importante se refiere al autocuidado relacionado con la sexualidad. En relación a esta, se deben observar tres aspectos importantes: las reacciones del cuerpo al tratamiento (disminución o no de la libido), el ejercicio de la sexualidad en este período (calidad y frecuencia de la erección o impotencia) y la identificación de los sentimientos experimentados por el paciente frente a todo esto. Los estudios revisados sobre los cuidados durante el tratamiento del CaP son dirigidos a la incontinencia urinaria y a la impotencia sexual, vale decir, a las consecuencias físicas más comunes en el tratamiento de la enfermedad.

No obstante, el mejor abordaje para el manejo del cáncer es el autocuidado. En un contexto más amplio, este término tam- bién refiere al compromiso del paciente con la observación, reconocimiento e identificación de síntomas, juzgando su calidad de vida y evaluando y adoptando estrategias más efectivas para promover comportamientos de autocuidado (Qian \& Yuan, 2012).

Otro aspecto importante tiene relación con la educación sobre la enfermedad, o sea, las informaciones transmitidas (orales y escritas) al paciente y al familiar. Esta estrategia es una importante herramienta y ayuda a la interacción con el profesional de la salud, permitiendo un mayor autocontrol del paciente y mejorando su autoconfianza. Asimismo, son observadas las necesidades individuales, la edad y la escolaridad, considerando que las consecuencias del tratamiento pueden tener mayor impacto en hombres más jóvenes.

El impacto en la función sexual, fundamentalmente, es observado en la dimensión física (alteración de la erección, calidad y frecuencia de la erección e impotencia completa) y, consecutivamente, acaba influyendo en la dimensión emocional. El enfrentamiento emocional de las consecuencias físicas termina por representar mayores situaciones de estrés frente a la falta de control sobre la vida, principalmente, en los hombres.

Estos aspectos influyen en la participación en el tratamiento, mantenimiento y adaptación al tratamiento oncológico y consecuentemente en el autocuidado, pues torna al hombre más vulnerable y frágil, condición que no se condice con su papel. Es importante resaltar que la participación de pacientes con cáncer en programas dirigidos al autocuidado causa cambios comportamentales y una mayor promoción de la salud (John, 2010) o, en otras palabras, una mayor calidad de vida.

En este contexto, son pocas las evidencias sobre los principales comportamientos de autocuidado que deben ser llevados a cabo en el post-tratamiento del CaP. En contrapartida, se estima una prevalencia (cinco años) de 3,8 millones de sobrevivientes de $\mathrm{CaP}$ en términos globales, con una previsión de aumento rápido en el futuro (Surbone \& Tralongo, 2016).

En el cáncer en general, se observan complicaciones cardiovasculares, cognitivas, neuropatías periféricas, síndromes de dolor, fatiga crónica, disfunción sexual e infertilidad (Ferro \& Borràs, 2011). Considerando que el paciente esté curado, estas consecuencias pueden surgir inmediatamente al término del tratamiento o a largo plazo.

Con relación al $\mathrm{CaP}$, se sugiere que el comportamiento de autocuidado debe ser semejante al que ocurre en otros tipos de cáncer. En este sentido, debe estar relacionado con las visitas médicas y el acompañamiento de los efectos secundarios de la enfermedad y del tratamiento (Castro \& Moro, 2012). Además, la prevalencia de las preocupaciones psicosexuales está presente en un rango que va, aproximadamente, del 30 al $50 \%$ de los sobrevivientes de CaP. Se destacan también la necesidad de información y la necesidad de soporte en los aspectos psicológicos y sexuales (Sourbone \& Tralongo, 2016).

Esta evidencia puede ser observada en la revisión integrativa sobre los cuidados de los pacientes en el post-operatorio de prostatectomía realizada por Santos, Silva, Saldanha, Lira \& Vítor (2012). En ella se destacan seis categorías principales: acompañamiento psicológico, orientaciones post-operatorias, tratamiento de la disfunción eréctil, tratamiento de la incontinencia 
urinaria, tratamiento del dolor y tratamiento de la hiponatremia. En este estudio, han sido identificadas recomendaciones de cuidados de enfermería centrados en el acompañamiento psicológico mediante acciones de apoyo emocional y de carácter educativo a los pacientes durante el período post-operatorio y el tratamiento de la disfunción eréctil.

En un estudio semejante, que también constituye una revisión integrativa de la literatura sobre medidas educativas adoptadas en el período de post-tratamiento de la prostatectomía, se han identificado problemáticas relacionadas con la sexualidad y las actitudes frente a los síntomas asociados al tratamiento y los efectos colaterales de la prostatectomía. No obstante, pocos estudios han abordado cuidados con el catéter de demora, prevención de infecciones, cuidados con la nutrición y la hidratación, retorno a las actividades, cuidados relativos a la higiene, orientación sobre ejercicios para la musculatura pélvica y control del dolor, considerados por los autores como fundamentales para una buena recuperación y autocuidado en el post-tratamiento. Para los autores, es importante un mayor número de medidas dirigidas a la orientación y a la capacitación del paciente en el desarrollo del su autocuidado, estimulando su empoderamiento (Rêgo, Lima, Fernandes, Saldanha, Lira \&Vitor, 2014).

En ambos estudios se percibe que el comportamiento de autocuidado deberá involucrar el auto-monitoreo de los síntomas físicos y psicológicos. Es importante resaltar que para los hombres, los síntomas físicos pueden ser observados de forma más natural, a diferencia de lo que ocurre en relación a los síntomas psicológicos, los cuales son más fácilmente ignorados en términos generales. De esta forma, se comprende el énfasis dado a los aspectos psicológicos y emocionales. Respecto a los mismos, se sugieren abordajes mediante estrategias de orientación y capacitación del paciente, con un objetivo educativo que permita desarrollar de forma efectiva su autocuidado, además de estimular un mayor control sobre su vida.

El énfasis en el impacto psicológico y en las posibles alteraciones emocionales que afectan a la población masculina después del tratamiento para el $\mathrm{CaP}$ ha sido el objetivo del estudio realizado por Oliveira, Santos, Rocha, Braga, Alves y Souza (2014). Los autores concluyen que factores de estrés y ansiedad son los aspectos más comunes en las evaluaciones de este público. Los autores utilizan escalas que, además de auxiliar en la mediación, se tornan un medio facilitador de la comunicación entre profesional y paciente.

Una estrategia de intervención innovadora en la mejoría del autocuidado y del control de síntomas son los programas de computadora con acceso on line o por teléfono. Al evaluar el efecto de una intervención en la educación y en la comunicación basada en coaching, realizada con 779 pacientes de cáncer (inclusive de $\mathrm{CaP}$ ) en diferentes fases del tratamiento, se ha demostrado que los pacientes han sido beneficiados por la misma, fundamentalmente, hombres de más de 50 años. Adicionalmente, los pacientes han presentado una disminución de los síntomas de angustia durante y después del tratamiento del cáncer (Berry et. al, 2014).

La discusión sobre el comportamiento de autocuidado en el post-tratamiento del $\mathrm{CaP}$, es semejante a la que ocurre en el tratamiento y presenta un mayor énfasis en la disfunción se- xual y en la incontinencia urinaria. A pesar de ello, se observa que, en esta fase, existe una preocupación mayor por las repercusiones emocionales que por las consecuencias de la enfermedad y tratamiento en los hombres. La orientación y capacitación (de forma oral y escrita), de cuño educativo, así como acciones de apoyo emocional, pueden ser estrategias que ayuden al autocuidado de los hombres.

Los estudios que abordan el autocuidado en los post-tratamiento de $\mathrm{CaP}$ y que abordan el punto de vista de los sobrevivientes de CaP (Ferro \& Borràs, 2011). De esta forma, se sugiere la necesidad de generar estudios centrados en los elementos que componen el proceso de salud/enfermedad/ cuidado de los hombres (Modena et al, 2013) así como el autocuidado, de forma más amplia. En este contexto, es importante promover estudios que integren la mirada de equipos interdisciplinarios y sobrevivientes del CaP. De este modo, se facilitarían una mejor y más amplia recopilación de evidencias, así como el abordaje de aspectos físicos, psicológicos, sociales, espirituales y dimensiones de la calidad de vida; todas ellas, características de suma importancia.

\section{Discusión}

El presente artículo tuvo como objetivo realizar una revisión integrativa de la literatura sobre los factores psicosociales relacionados con el comportamiento de autocuidado de los hombres para la prevención, tratamiento y post-tratamiento del cáncer de prostata. El CaP es una enfermedad muy frecuente en la población masculina, no obstante, es poco conocida por los hombres, así como los exámenes preventivos que permiten su diagnóstico en la fase inicial y, consecuentemente, un buen pronóstico.

Considerada una enfermedad que tiene un gran impacto en la masculinidad (debido a la localización anatómica), se presenta con potencial para desencadenar problemas relacionados con la sexualidad. No obstante, se observa una resistencia en el público masculino a asumir comportamientos de autocuidado en su prevención, así como en su tratamiento y posttratamiento.

El autocuidado relacionado con la prevención del CaP es esencial, aún más con pocas medidas preventivas específicas. Su etiología es considerada indeterminada y los síntomas poco claros para que el individuo pueda reconocer e identificar la enfermedad en las fases iniciales. Por lo tanto, es importante diseminar los principales factores de riesgo que, en el caso del $\mathrm{CaP}$, están relacionados con la existencia de antecedentes familiares (padre y hermano), la etnia (mayor riesgo para hombres de raza negra y latinoamericanos) y la edad. Estas informaciones son de gran importancia, pues permiten al público masculino incrementar su conocimiento sobre el riesgo de sufrir la enfermedad.

El autocuidado en la prevención del CaP está relacionado con la búsqueda de información y la realización de los exámenes de prevención PSA y/o ETR. Se percibe en la literatura una variedad de estudios relacionados con el conocimiento de la enfermedad, los exámenes preventivos y la intención de realizarlos. Los resultados encontrados son preocupantes, pues la mayoría demuestra el poco conocimiento que los hombres tienen sobre los mismos y su relación con la prevención del CaP. En el caso del ETR, las evidencias presentadas sugieren 
la poca adhesión del público masculino a este examen, considerándolo embarazoso e incómodo. A pesar de ello, el ETR es el examen más indicado para la detección del CaP. De este modo, se hace evidente la necesidad de promover de forma más amplia la diseminación de informaciones sobre el CaP y los exámenes para su prevención (principalmente el ETR) en consultorios médicos, centros de salud, hospitales y empresas que tengan acceso al público masculino. Estrategias como esta pueden generar en los hombres una mayor consciencia sobre la gravedad de esta enfermedad, el riesgo personal de sufrirla, así como fomentar una mayor responsabilidad sobre la propia salud, a través de comportamientos de autocuidado efectivos en la prevención del CaP.

Intervenciones psicosociales, educativas e interdisciplinarias pueden ser estrategias importantes para el desarrollo del autocuidado en el género masculino. Es evidente que hombres y mujeres tienen necesidades diferentes. Mediaciones con un equipo interdisciplinario facilitarían la identificación de las singularidades del proceso salud-enfermedad en el género masculino que permitirían intervenciones más efectivas para el desarrollo de nuevos estándares de comportamiento y actitudes relacionadas con el autocuidado.

Asimismo, estas acciones precisan ser incorporadas a los tres niveles de atención, pero principalmente a la atención primaria, responsable de las medidas preventivas específicas para el autocuidado. También se hace necesario incluir acciones educativas de salud, a través de la producción de campañas y acciones de sensibilización que no busquen solo la información, sino que auxilien la discusión y construcción de estrategias que fomenten el autocuidado en el género masculino. Cabe destacar la constante necesidad de capacitación de los profesionales en la orientación y atención al público masculino, fundamentalmente, en la atención básica. En este sentido, contribuir con una mayor calidad de asistencia en la prestación de servicios podrá disminuir la distancia entre los hombres y los servicios de salud, esencialmente, con los profesionales de la salud. Estas medidas pueden propiciar acciones preventivas más efectivas, mayor adhesión a los programas y servicios específicos para la población masculina y, consecuentemente, posibilitarán un mayor autocuidado.

Al tener un diagnóstico de $\mathrm{CaP}$, el hombre puede vivir una fase de fragilidad e impotencia delante de la nueva condición, que exigirá cambios significativos desde la identidad hasta el ejercicio de la masculinidad. La atención a los aspectos emocionales debe ser constante, pues en este periodo se dará el ajuste a la enfermedad y el proceso de adaptación que auxiliará los cambios necesarios en las actividades básicas e instrumentales de vida cotidiana.

El autocuidado en la fase de tratamiento es de gran importancia para el género masculino. En relación al CaP, emerge como notoria la falta de conocimiento de la enfermedad, la cual puede dificultar la administración de la condición de enfermo, exigiendo una mayor atención por parte de los profesionales de la salud y de la familia. En este sentido, los estudios focalizados en la fase de tratamiento del CaP enfatizam estrategias educativas y de auto-monitoreo.

Algunas de las consecuencias más comunes en el tratamiento como lo son la incontinencia urinaria, la impotencia sexual y la pérdida de la libido pueden generar sentimientos de ansie- dad, miedo, angustia, tristeza, incertidumbre, incapacidad y sensación de vacío. A su vez, estos pueden influenciar directamente el comportamiento de autocuidado. Dado el caso, es necesario que el paciente adopte un comportamiento activo en el reconocimiento e identificación de los síntomas físicos y psicológicos mediante la observación y el auto-monitoreo, buscando estrategias que puedan ser evaluadas según su eficiencia para el autocuidado en esta fase. Estas estrategias ayudan a hacer frente a los efectos adversos de la enfermedad y disminuyen el impacto en su vida.

Pocos estudios son realizados con los sobrevivientes de este tipo de cáncer. No obstante, las tasas de sobrevivencia están aumentando en los últimos años. Al adoptar comportamientos de autocuidado desde el tratamiento, se presupone que el paciente con CaP mejorará su aptitud para enfrentar las consecuencias del post-tratamiento y su atención a los efectos del tratamiento (efectos primarios) y post-tratamiento (efectos secundarios). En la fase del tratamiento y debido a su influencia directa en la sexualidad, se manifiestan con mayor énfasis aspectos emocionales. Ello es debido a que la sexualidad es considerada por el hombre como central en el ejercicio de su masculinidad. Al retornar a las actividades realizadas anteriormente a la enfermedad, el comportamiento de autocuidado deberá ayudar a un enfrentamiento más activo a las posibles secuelas físicas, emocionales y sociales.

El CaP para los hombres es una condición de gran impacto en su vida. Las conductas de autocuidado son esenciales para la prevención y la aceptación de la condición de enfermo. Se percibe que, para el público masculino, la experiencia de la enfermedad puede representar cambios significativos no solo en su vida, sino en el papel social de hombre y en el ejercicio de la masculinidad. Es necesario que los profesionales de la salud, principalmente los psicólogos, participen de forma activa en todas las etapas de la enfermedad para poder realizar intervenciones que refuercen la recuperación de la autonomía y complementen los comportamientos de autocuidado en el público masculino.

\section{Referencias}

Addis, M. E. \& Mahalik, J.R. (2003). Men, masculinity, and the contexts of help seeking.

American Psychologist, 58(1), 5-14.http://dx.doi.or$\mathrm{g} / 10.1037 / 0003-066 \mathrm{X} .58 .1 .5$

Alves, R.F.; Silva, R.P.; Ernesto, M.V.; Lima, A.G.B. \& Souza, F.M. (2011). Gênero e saúde: o cuidar do homem em debate. Psicologia: Teoria e Prática.13(3):152-166.

Arruda, G.O.; Mathias, T.A.F. \& Marcon, S.S. (2017). Prevalence and factors associated with the use of public health services for adult men. Ciência \& Saúde Coletiva. 22(1):279-290. http://dx.doi.org/10.1590/141381232017221.20532015

Astudillo, M.N.M.; Pinzón, L.A.S.; Ospina, J.J., Grisales, A. \& García, J.D.R. (2011, jul- dez).Percepciones sobre el câncer de próstata em población masculina mayor de 45 años. Santa Rosa de Cabal, 2010. Hacia la Promoción de la Salud. 16(2): 147-161.

Barberá, E. \& Benlloch (coords). Psicología y género PEARSON EDUCACIÓN, S. A., Madrid, 2004.

Belinelo, R.G.S.; Almeida, S.M.; Oliveira, P.P. de; Onofre, P.S. de C.; Viegas, S.M da F. \& Rodrigues, A.B. (2014). Exames de rastreamento para o câncer de próstata: 
vivência de homens. Escola Anna Nery Revista de Enfermagem.18 (4):697-704.

Bennet, G. \& Badger, T.A. (2005, may). Depression in men with prostate cancer. Oncolgy Nursing Forum. 32(3):545-556. Doi:10.1188/05.ONF.545-556

Berry, D.L. et. al, (2013). Electronic Self-Report Assessment for Cancer and Self-Care

Support: Results of a Multicenter Randomized Trial. Journal of Clinical Oncology, 32:199-205. DOI: $10.1200 / J C O .2013 .48 .6662$

Bolster D.; Manias, E. (2010). Person-centered interactions between nurses and patients during medication activities in an acute hospital setting: Qualitative observation and interview study. Intentional Journal of Nursing Studies; 47:154-65. doi:10.1016/j.ijnurstu.2009.05.021

Bratt, (2002). Hereditary prostate câncer: clinical aspects. The Journal of Urology. (168): 906 -91. https://doi. org/10.1016/S0022-5347(05)64541-7

Carneiro, L. M. R.; Santos, A., Paz, M.; Macena, R.H.M. \& Vasconcelos, T.B. (2016).Atenção Integral à saúde do homem: desafio na atenção básica. Revista Brasileira em Promoção da Saúde: 29(4):554-563.

Castro, E. K. \& Moro, L. (2012). Factores psicosociales relacionados com el autocuidado em la prevención, tratamiento y postratamiento del câncer de mama. Psicoconcología. 9 (2-3):453-465.

Chan, E.C.Y. et. Al. (2011).A community-based intervention to promote informed decision making for prostate cancer screening among Hispanic American men changed knowledge and role preferences: A cluster RCT. Patient Education and Counseling. 84:4461.

Cooperberg, M.R. \& Chan, J.M. (2017). Epidemiology of prostate cancer. World Journal Urology, 35:849 DOI 10.1007/s00345-017-2038-0

Couto, M.T. et al. (2010). Men in primary healthcare: discussing (in) visibility based on gender perspectives. Interface: Comunicação, Saúde e Educação, 14(33):25770.

Courtnay W.H. \& Keeling R. P. (2000). Men, gender, and health: toward an interdisciplinary approach. Journal of American College Health. 48(6):243-6. DOI: $10.1080 / 07448480009596265$

Courtenay W.H. (2000). Constructions of masculinity and their influence on men's well- being: a theory of gender and health. Social Science \& Medicine. 50(10):1385401. DOI:10.1016/S0277-9536(99)00390-1

Costa-Júnior, F.M. da \& Maia, A.C.B. (2009). Concepções de homens hospitalizados sobre a relação entre gênero e saúde. Psicologia: Teoria e Pesquisa. 25(1):55-63.

Damião, R.; Figueiredo, R.T.; Dornas, M.C.; Lima, D.S. \& Koschorke, M.A.B. (2015, agos). Câncer de Próstata. Revista do Hospital Universitário Pedro Hernesto. 14(supl.1):80-86.

Escurriola, M.F.; Font, L.G.; Forès, R.A.; Brunet, J.C. \& Torrens, M.B. (2008). Conocen los varones de 50-70 años la efectividad, los beneficios y los riesgos del cribado del cáncer de próstata? Atención Primaria. 40(7): 357-61. https://doi.org/10.1157/13124129

Fernandes, M.V.; Martins, J.T.; Cardelli, A.A.M.; Marcon, S.S. \& Ribeiro, R.P. (2014). Perfil epidemiológico do homem com câncer de próstata atendido em um hospital universitário. Cogitare Enfermagem. 19(2):330-40.

Ferro, T. \& Borràs, J.M. (2011). Una bola de nieve está creciendo en los servicios sanitarios los pacientes supervivientes de câncer. Gaceta Sanitaria. 25(3):240245. doi:10.1016/j.gaceta.2010.12.002

Galvão, M.T.R.L.S. \& Janeiro, J.M.S.V. (2013). O autocuidado em enfermagem: autogestão, automonitoração e gestão sintomática como conceitos relacionados. Reme: Revista Mineira de Enfermagem. 17(1):225230. DOI: $10.5935 / 1415-2762.20130019$

Gomes, R.; Nascimento, E.F. de \& Araújo, F.C. de (2007). Por que os homens buscam menos os serviços de saúde do que as mulheres? As explicações de homens com baixa escolaridade e homens com ensino superior. Cadernos de Saúde Pública. 23(3):565-574.

Guedes, G.A. \& Caldeira, A.M.Jr. (2014, jan-jun). As vantagens da utilização do exame antígeno prostático específico (PSA) no diagnóstico do câncer de próstata. Revista de Divulgação Científica Sena Aires. (1):8996.

Hayes B. \& Bonner A. \& Pryor J. (2010) Factors contributing to nurse job satisfaction in the acute hospital setting: A review of recent literature. Journal Nursing Management, 18:804-14. DOI: 10.1111/j.13652834.2010.01131.x

Hasanpour-Dekordi, A. (2016). Self-care Concept Analysis in Cancer Patients: An Evolutionary Concept Analysis. Indian Journal of Palliative Care. 22(4):388-394

Instituto Nacional do Câncer (INCA). http://www2.inca.gov. $\mathrm{br} / \mathrm{wps} / \mathrm{wcm} / \mathrm{connect} /$ inca/portal/home

John L.D. (2010). Self-care strategies used by patients with lung cancer to promote quality of life. Oncology Nursing Forum, 37:339-47. DOI:10.1188/10.ONF.339347

Junior, C.V.X. \& Hachul, M. (2014). Tumores urológicos no Brasil. Revista Brasileira de Medicina .71 (11), p. 410-414.

Mata, L.R.F \& Napoleão, A.A. (2010). Intervenções de enfermagem para alta de paciente prostatectomizado: revisão integrativa. Acta Paulista de Enfermagem. 23(4):574-9.

Martins, A.M. \& Gazzinelli, A.P.; Almeida, S.S.L de \& Modena, C.M. (2012).Concepções de psicólogos sobre o adoecimento de homens com câncer. Psicologia: Teoria e Prática. 14(2):74-87.

Mestrino, B. V.; Gomes, L., Almeida, J.L.T. de; Almeida, J.C. de; \& Oliveira, R.V.L. de. (2011). Does clarifying the digital rectal examination to the elderly reduce the discomfort in its first execution? Revista Col. Brasileira Cir. 38(6): 407:4011.

Miranda, P.S.C.; Còrtes, M. da C.J.W.; Martins, M.E.; Chaves, P.C. \&Santarosa, R.C. (2004). Práticas de diagnóstico precoce de câncer de próstata entre professores da Faculdade de Medicina - UFMG. Revista da Associação Médica Brasileira.50(3):272- 275.

Modena, C.M.; Martins, A.M.; Ribeiro, R.B.N. \& Almeida, S.S.L. de (2013, Jul-Set). Os homens e o adoecimento por câncer: um olhar sobre a produção científica brasileira. Revista Baiana de Saúde Públic. 37(3): 644-660.

Moreira, M.C.N.; Gomes, R. \& Ribeiro, C.R. (2016). E agora o homem vem? Estratégias de atenção à saúde dos homens. Cadernos de Saúde Pública, 32(4):0060015, ab http://dx.doi.org/10.1590/0102-311X00060015 
Moscheta, M. dos S. \& Santos, M. A. (2012). Grupos de apoio para homens com câncer de próstata: revisão integrativa da literatura. Ciência e Saúde Coletiva: 17(5):1225-1 National Cancer Institute (NIH). https://www.cancer.gov/

Oliveira et al., (2014). Aspectos emocionais pós-tratamento do câncer de próstata: uma revisão integrativa da literatura. Online Brasilian Journal oj Nursing. 699-707 http://dx.doi.org/10.17665/1676-4285.2014v13n4

Pedersen, V.H.; Armes, J. \& E. Rean (2012). Perceptions of prostate cancer in Black African and Black Caribbean men: a systematic review of the literature. Psycho-Oncology 21: 457-468.

Pedrosa, N.T. \& Polejack, L. (2016, Jul-Dez). Cuidado e autocuidado em oncologia: significados para profissionais e usuários. Mudanças - Psicologia da Saúde. 24 (2): 1-10.

Perea, M.D.; Castaño-Vinyalsb, G.; Altzibare, J.M.; Ascuncef, N.; Moreno, V.; Tardonh, A.; Polláni, M.; Salaa, M. (2012).Prácticas de cribado de cáncer y estilos de vida asociados e la población de controles del estudio espa nol multi-caso control (MCC-Spain). Gaceta Sanitária, 26(4): 301-310. doi:10.1016/j.gaceta.2012.01.020

Pinquart M. \& Duberstein, P.R. Depression and cancer mortality: A meta-analysis. Psychol Med 2010; 40:1797-810. doi: 10.1017/S0033291709992285.

Poonnappa, B.G. (2014). Men's Health: A Different Perspective. American Journals Men's Health, JIMSA, 27(4).

Pozzati, R.; Beuter, M.; Rocha, L.S.; Santos, N.O.; Budó, M. de L.D. \& Perlini, N.M.O.G. (2013). O cuidado da saúde dos homens: realidadese perspectivas. Revista de Enfermagem - UERJ, 21(4):540-545.

Qian H., Yuan C. (2012) Factors associated with self-care self-efficacy among gastric and Colorectal cancer patients. Cancer Nursing.35:E22-3

Santos, D.R.F. dos; Silva, F.B.L. e; Saldanha, E. de A.; Lira, A.L.B. de C. \& Vitor, A.F. (2012, jul/sep). Cuidado de enfermagem ao paciente pós-operatório de prostatectomia: revisão integrativa. Revista Eletrônica de Enfermagem. 14(3): 690-701.

Separavich, M.A. \& Canesqui, A.M. (2013). Saúde do homem e masculinidades na Politica Nacional de Atenção Integral à Saúde do Homem: uma revisão bibliográfica. Saúde e Sociedade São Paulo. 22(2):415428.

Silva et al (2008). Cuidado, autocuidado e cuidado de si: uma compreensão paradigmática para o cuidado de enfermagem. Revista Esc. Enfermagem (USP). 43(3): 697-703.

Silva, F.A. \& Silva, I.R. de (2014). Sentidos de saúde e modos de cuidar de si elaborados por homens usuários de Unidade Básica de Saúde - UBS. Ciência \& Saúde Coletiva. 19(2):417- 428.

Schwarz, E.; Gomes, R.; Couto, M.T.; Moura, E.C. de; Carvalho, S. de A. \& Silva, S. F. C de. (2012, dez). Política de Saúde do Homem. Revista Saúde Pública. 46 (Supl): 108-116. http://dx.doi.org/10.1590/S003489102012005000061

Sørensen K. et. al, (2012).Health literacy and public health: A systematic review and integration of definitions and models. BMC Public Health, 12:80. https://doi. org/10.1186/1471-2458-12-80
Surbone A, Tralongo P. (2016). Categorization of cancer survivors: why we need it. Journal of Clinical Oncology, 34(28):3372-3374. DOI: 10.1200/JCO.2016.68.3870;

Rego et al., (2014). Educational steps adopted in post-operative period of prostatectomy: an integrative review. Journal Nurse UFPE. 8 (suplem.3):4122-30.

Tonon, T.C.A. \& Schoffen, J.P.F. (2009, set-dez). Câncer de prostate: uma revisão da literatura. Revista Saúde e Pesquisa.2(3):403-410.

Zacchi, S.R.; Amorin, M. H.C.; Souza, M.A. C. de; Mitto, M.H.M. de B. \& Zandonade, E. (2014). Associação de variáveis sociodemográficas e clínicas com o estadiamento inicial em homens com câncer de próstata. Cadernos de Saúde Coletiva. 22(1): 93-100.

Wikimon, A. \& Whitehead, L. (2009). Evolution of the concept $f$ self-care and implications for nurses: a literature review. International journal of Nursing Studies. 46:1143-1147.

Wilde, M.H. \& Garvin, S. (2007). A concept analysis of self-monitoring. Journal Advance Nursing. 57:339-50. DOI: 10.1111/j.1365-2648.2006.04089.x

Willians, B. \& Pace, A.B. (2009). Problem based learning in chronic disease management: a review of the research. Patient Education and Counseling. 77(1):1419. https://doi.org/10.1016/j.pec.2009.03.004

World Health Organization. Men, ageing and health. Geneva: World Health Organization; 2001. 